INPLASY

PROTOCOL

To cite: Zhou et al. Efficacy and safety of Chinese herbal medicine Xiao Yao San in polycystic ovary syndrome: a systematic review and metaanalysis. Inplasy protocol

202150031. doi:

10.37766/inplasy2021.5.0031

Received: 08 May 2021

Published: 08 May 2021

Corresponding author:

Yaping Wang

ypwang@jnu.edu.cn

Author Affiliation:

Formula-Pattern Research Center, School of Traditional Chinese Medicine, Jinan

University.

Support: ZH2020KF01; No. 2020B1111100001.

Review Stage at time of this submission: Preliminary searches.

Conflicts of interest:

None declared.

\section{Efficacy and safety of Chinese herbal medicine Xiao Yao San in polycystic ovary syndrome: a systematic review and meta-analysis}

Zhou, X1; Ma, QY2; Wang, YX3; Yan, ZQ4; Wang, YN5; Lin, SQ6; Chen, JX7; Wang, YP8.

Review question / Objective: 1. Are the Chinese herbal medicine, Xiao Yao San, effective in treating polycystic ovary syndrome? 2. Is it safe to use Xiao Yao San for the treatment of polycystic ovary syndrome?

Condition being studied: Polycystic ovary syndrome is characterised by anovulation, infertility, and hyperandrogenism, with clinical manifestations of irregular menstrual cycles, hirsutism, and acne. The condition affects an estimated $5-10 \%$ of women of reproductive age, although this varies depending on the diagnostic criteria used. One of the commonest presenting complaints of women with polycystic ovary syndrome is anovulatory infertility. They also have increased prevalence of cardiovascular risk factors similar to that seen in the metabolic syndrome.

INPLASY registration number: This protocol was registered with the International Platform of Registered Systematic Review and Meta-Analysis Protocols (INPLASY) on 08 May 2021 and was last updated on 08 May 2021 (registration number INPLASY202150031).

\section{INTRODUCTION}

Review question / Objective: 1. Are the Chinese herbal medicine, Xiao Yao San, effective in treating polycystic ovary syndrome? 2. Is it safe to use Xiao Yao San for the treatment of polycystic ovary syndrome?

Condition being studied: Polycystic ovary syndrome is characterised by anovulation, infertility, and hyperandrogenism, with clinical manifestations of irregular 
menstrual cycles, hirsutism, and acne. The condition affects an estimated $5-10 \%$ of women of reproductive age, although this varies depending on the diagnostic criteria used. One of the commonest presenting complaints of women with polycystic ovary syndrome is anovulatory infertility. They also have increased prevalence of cardiovascular risk factors similar to that seen in the metabolic syndrome.

\section{METHODS}

Participant or population: Women patients with polycystic ovary syndrome who attain Xiao Yao San or modified Xiao Yao san alone or with western medicines, will be included. No restriction on age, or nationality.

Intervention: Trials that compared Xiao Yao San or modified Xiao Yao San as the active intervention in the treatment group versus placebo or conventional medicines will be included. Trials will be excluded if any other medications, including qigong, Tai Chi, acupuncture, cupping, moxibustion, and massage, were used as co-interventions.

Comparator: Placebo or conventional medicines for polycystic ovary syndrome.

Study designs to be included: Randomized controlled trials.

Eligibility criteria: Inclusion criteria Studies were eligible for inclusion if they met all of the following five criteria: (1) patients were diagnosed with polycystic ovary syndrome either by a clinician or according to specific diagnostic criteria; (2) studies were conducted as RCTs; (3) effects of XYS or modified XYS (MXYS) in treating polycystic ovary syndrome were assessed; (4) the possible comparisons were as follows: XYS or MXYS vs. placebo, XYS or MXYS plus conventional medicines vs. conventional medicines; XYS or MXYS plus conventional medicines vs. placebo; (5) efficacy evaluation criteria were sufficiently described. Exclusion criteria Studies involving post-menopausal women (over 50 years of age) were excluded.
Information sources: Comprehensive search will be carried out in seven electronic databases, including MEDLINE, Embase, the Cochrane Library, Chinese Biomedical Database (CBM), China National Knowledge Infrastructure (CNKI), Wanfang Database, China Science and Technology Journal Database (VIP). No publication date or language restriction will be imposed.

Main outcome(s): Clinical outcomes: ovulation rate.

Additional outcome(s): Clinical pregnancy and live births, weight, blood pressure and adverse effect; biochemical outcomes: hormone, insulin and lipids.

Data management: Selection of studies: Two reviewers will independently screen the titles, abstracts and manuscripts retrieved from different databases engines and identify studies that potentially meet the inclusion criteria outlined above. Study selection and exclusion process will be documented and a list of potentially eligible studies in the analysis will be created. The full text of these potentially eligible studies will be downloaded and further assessed for eligibility by the two reviewers. Any disagreements will be resolved by a third reviewer as necessary. The reasons for the exclusion of any studies will be recorded on a PRISMA flow chart. Data extraction: Data extraction will be performed by two independently reviewers using a predesigned collection form. If any discrepancies arise, an additional reviewer will be consulted. The following information will be extracted for each study: (1) Publication characteristics; (2) Participants characteristics; (3) Interventions: intervention, comparison, concomitant medications, duration of treatment and follow-up time. (4) Outcomes: primary and secondary outcomes and adverse events.

Quality assessment / Risk of bias analysis: The methodological quality of each study was assessed by Dr. Xuan Zhou, Wang Yaxin, and Yan Zhenqian according to criteria from the Cochrane risk of bias, including random sequence generation 
(selection bias), allocation concealment (selection bias), blinding of participants and personnel (performance bias), blinding of outcome assessment (detection bias), incomplete outcome data (attrition bias), selective reporting (reporting bias) and other bias. Any discrepancies will be resolved by Dr. Wang.

Strategy of data synthesis: Statistical analyses will be performed using the Cochrane RevMan software (version 5.3.0, Copenhagen, the Nordic Cochrane Center). Dichotomous data will be presented as relative risk (RR) or odds ratio (OR) with 95\% confidence intervals (Cls). The $X^{2}$ test will be used to evaluate the heterogeneity and $I^{2}$ will be used to assess the inconsistency across studies. Values of $\mathbf{I}^{2}$ ranged from 0 to $100 \%\left(I^{2}<40 \%\right.$, might not be important; $30 \%<\mathrm{I}^{2}<60 \%$, moderate heterogeneity; $50 \%<I^{2}<90 \%$, substantial heterogeneity; $75 \%<I^{2}<100 \%$, considerable heterogeneity). Fixed-effect model will be used to pool estimates. Potential sources of heterogeneity will be identified by subgroup analysis and sensitivity analysis. Potential publication bias will be assessed graphically with funnel plot.

Subgroup analysis: Subgroup analyses will be conducted according to interventions in trial group.

Sensitivity analysis: Sensitivity analysis will be undertaken to examine if the effects are modified by, or robust to, the type of measurement method or tool.

Language: No restriction.

Country(ies) involved: China.

Keywords: Chinese herbal medicine; Xiao Yao San; polycystic ovary syndrome; metaanalysis.

Contributions of each author:

Author 1 - Xuan Zhou.

Author 2 - Qingyu Ma.

Author 3 - Yaxin Wang.

Author 4 - Zhenqian Yan.

Author 5 - YaNan Wang.
Author 6 - Shaoqin Lin.

Author 7 - Jiaxu Chen.

Author 8 - Yaping Wang. 